\title{
Corrigendum
}

\section{Corrigendum to "Anti-Inflammatory Effect of Geniposide on Osteoarthritis by Suppressing the Activation of p38MAPK Signaling Pathway"}

\author{
Yuan Chen, ${ }^{1}$ Kangquan Shou $\mathbb{D},{ }^{2}$ Chunlong Gong, ${ }^{2}$ Huarui Yang $\mathbb{D},{ }^{2}$ Yi Yang $\mathbb{D},{ }^{2}$ \\ and Tongzhu Bao ${ }^{2}$ \\ ${ }^{1}$ Department of Emergency and ICU, The First Clinical Medical College, China Three Gorges University, Yichang, \\ Hubei 443002, China \\ ${ }^{2}$ Department of Orthopaedics, The First Clinical Medical College, China Three Gorges University, Yichang, Hubei 443002, China \\ Correspondence should be addressed to Tongzhu Bao; baotongzhu110@163.com
}

Received 17 December 2021; Accepted 17 December 2021; Published 10 January 2022

Copyright (c) 2022 Yuan Chen et al. This is an open access article distributed under the Creative Commons Attribution License, which permits unrestricted use, distribution, and reproduction in any medium, provided the original work is properly cited.

In the article titled "Anti-Inflammatory Effect of Geniposide on Osteoarthritis by Suppressing the Activation of p38MAPK Signaling Pathway" [1], an image duplication was identified in Figure 5. The authors explained that this was due to an error introduced during the preparation of the manuscript and provided the correct panels as below. 

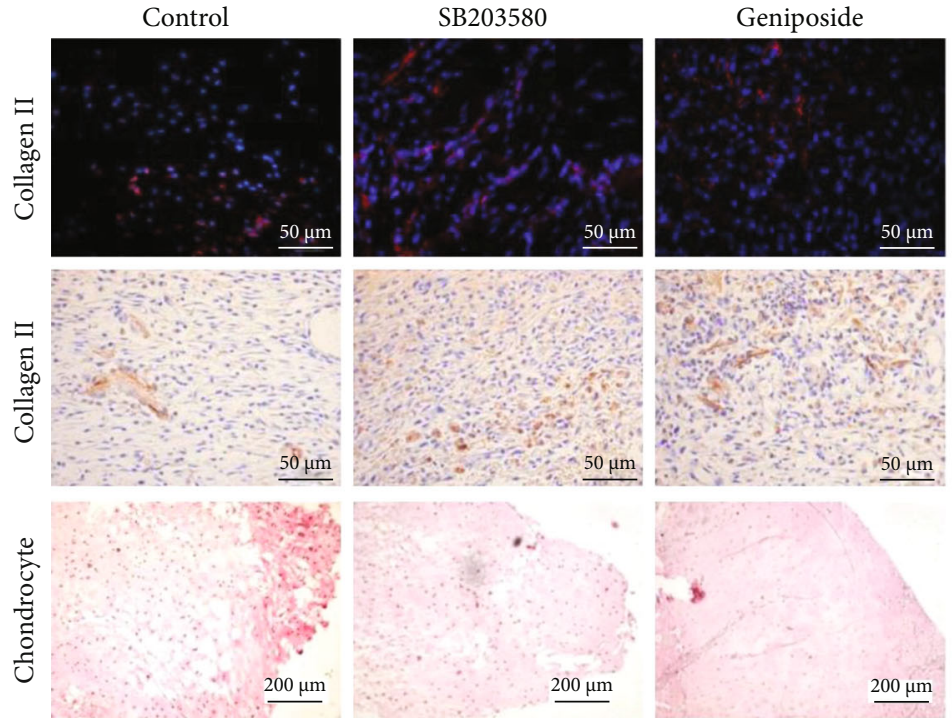

Geniposide+SB203580
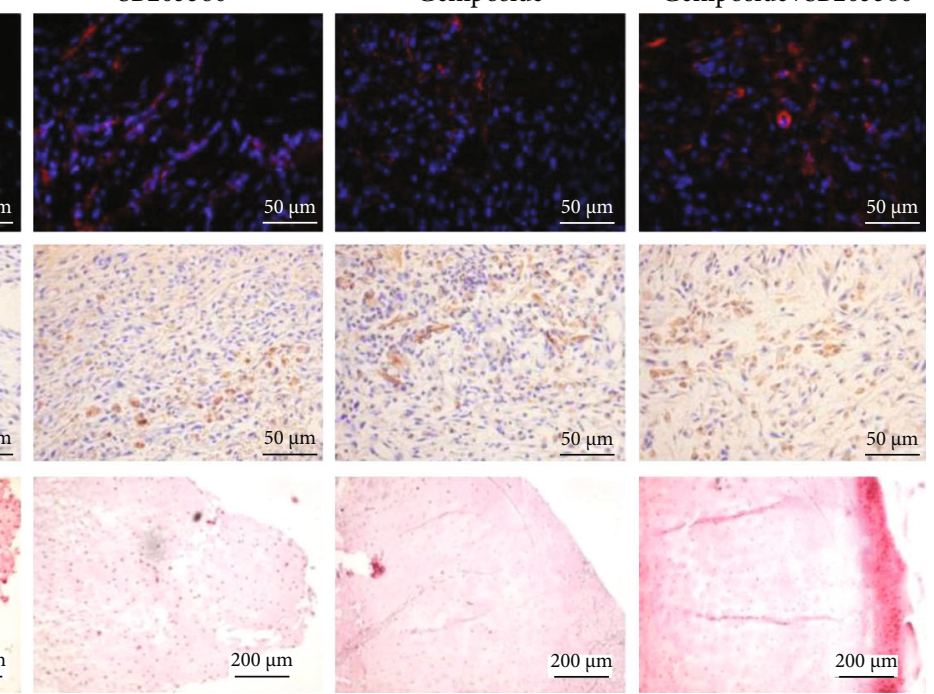

Figure 5

\section{References}

[1] Y. Chen, K. Shou, C. Gong, H. Yang, Y. Yang, and T. Bao, "AntiInflammatory Effect of Geniposide on Osteoarthritis by Suppressing the Activation of p38MAPK Signaling Pathway," BioMed Research International, vol. 2018, Article ID 8384576, 11 pages, 2018. 